\title{
Joint Time Delay and Angle Estimation Using the Matrix Pencil Method Based on Information Reconstruction Vector
}

\author{
Haiwen $\mathrm{Li}^{1,2^{*}}$, Xiukun Ren ${ }^{1}$, Ting Bai ${ }^{1}$, and Long Zhang ${ }^{1}$ \\ 1China National Digital Switching System Engineering and Technological Research Center \\ Zhengzhou, Henan, 86-450002, China \\ 2 Army Engineering University Communication Sergeant School \\ Chongqing 86-400035, China \\ [e-mail: luckylhw66@163.com] \\ *Corresponding author: Haiwen Li
}

Received March 2, 2018; revised June 7, 2018; accepted July 18, 2018; published December 31, 2018

\begin{abstract}
A single snapshot data can only provide limited amount of information so that the rank of covariance matrix is not full, which is not adopted to complete the parameter estimation directly using the traditional super-resolution method. Aiming at solving the problem, a joint time delay and angle estimation using matrix pencil method based on information reconstruction vector for orthogonal frequency division multiplexing (OFDM) signal is proposed. Firstly, according to the channel frequency response vector of each array element, the algorithm reconstructs the vector data with delay and angle parameter information from both frequency and space dimensions. Then the enhanced data matrix for the extended array element is constructed, and the parameter vector of time delay and angle is estimated by the two-dimensional matrix pencil (2D MP) algorithm. Finally, the joint estimation of two-dimensional parameters is accomplished by the parameter pairing. The algorithm does not need a pseudo-spectral peak search, and the location of the target can be determined only by a single receiver, which can reduce the overhead of the positioning system. The theoretical analysis and simulation results show that the estimation accuracy of the proposed method in a single snapshot and low signal-to-noise ratio environment is much higher than that of Root Multiple Signal Classification algorithm (Root-MUSIC), and this method also achieves the higher estimation performance and efficiency with lower complexity cost compared to the one-dimensional matrix pencil algorithm.
\end{abstract}

Keywords: Orthogonal frequency division multiplexing (OFDM), Matrix Pencil (MP), Time of Arrival (TOA), Angle of Arrival (AOA)

This work is supported in part by the National Natural Science Foundation of China (Grant No. 61401513) and the National Science and Technology Major Project of the Ministry of Science and Technology of China (Grant No. 2013ZX03006003-006). Grateful acknowledgement is made to the reviewers for suggesting ways to improve the readability of the paper. 


\section{Introduction}

In the actual signal environment, the fast moving target requires high real-time performance of the positioning system. The valid data received is only single snapshot data or several snapshot data, and the parameter estimation algorithm based on a large number of snapshot data can no longer meet the actual needs. Therefore, using single snapshot data to achieve the positioning parameters estimation becomes a solution.

Owing to the significant advantages of high spectrum efficiency, good anti-multipath fading characteristics and low implementation complexity, OFDM technology has been widely used in IEEE 802.11a/g/n/ac, OFDM new system radar [1], satellite communication system [2], WiMAX system, 3GPP LTE/LTE-Advanced and 5G mobile communication system [3], etc. When people enjoy data services provided by OFDM technology, the demand for wireless target location information is also increasing. Therefore, using OFDM array antenna receiving system to achieve accurate positioning of the target signal has become the current research hotspot.

Time of arrival (TOA) estimation of the target signal is a common range finding method in wireless location [4-6]. For time delay estimation of OFDM signal, three approaches, namely peak detection, modified maximum peak-to-leaking rate detection and channel frequency response (CFR) reconstruction are proposed in [7], and in WINNER A1 LOS channel, the CFR reconstruction method can achieve the best performance, but this method is mainly based on the received preamble sequence. The time difference of arrival (TDOA) estimation algorithm combining coarse estimation and fine estimation is presented in [8], the algorithm needs to use the technology of guided along detection to suppress multipath propagation, but the positioning accuracy is limited. Besides, Makki [9] proposes a TOA estimation algorithm of a coarse estimation using cross-correlation method and fine estimation using subcarrier phase difference, however this algorithm can only be used in the active position, and need the multi-receivers, which increases the system overhead. At the same time, if the array antenna can be used to obtain the signal angle of arrival (AOA) for joint estimation [10]-[11], then a receiver can determine the location of the target source, which can effectively reduce the positioning system overhead. Zhou [12] provides a joint estimation algorithm of delay and angle for OFDM signal, which uses the frequency domain data of single antenna to achieve MUSIC time delay estimation, and then use the array elements for MUSIC AOA estimation, but it fails to give a matching method of delay and angle, and its computational complexity is high. Ba [13] presents a joint estimation of delay and angle using 2D-MUSIC for two-dimensional searching, which solves the pairing problem, but the computational complexity is very high, and it is hard to be applied practically. The above algorithms all require large sample data, and can obtain excellent estimation performance in slow time-varying and multiple snapshots circumstances. This is because the classical subspace class super-resolution algorithms (such as MUSIC algorithm, ESPRIT algorithm) require that the rank of the covariance matrix of the received data is equal to the number of array elements, i.e., the number of snapshots is larger than the number of array elements. The accuracy of the estimation is based on the high precision signal subspace or noise subspace obtained from a large number of snapshot data. However, in practical application, especially when dealing with the short-time burst signal or fast moving target signal, only a single snapshot is available. For single snapshot data, the rank of the covariance matrix is 1 . Due to the rank loss of the covariance matrix, the performance of time delay estimation declines sharply. Generally, the spatial smoothing technology is adopted to make the covariance matrix full rank, but it needs 
array elements and sacrifices bandwidth. Therefore, the performance of parameter estimation is also affected.

The Matrix Pencil (MP) algorithm [14] can directly process the received signal data without calculating the covariance matrix of the signal. It has the advantage of super-resolution and fast computation speed, and it can estimate the location parameters only by single snapshot. At the same time, for the coherent signals in multipath environment, the direction of arrival (DOA) estimation [15] can be completed without using the spatial smoothing. But the shortcoming is the high demand for the signal to noise ratio. The one-dimensional (1D) MP algorithm is used to complete TDOA estimation and study the relationship between system bandwidth and the estimation error in [16]. The algorithm of matrix enhancement and matrix pencil in [17] is proposed to estimate two-dimensional (2D) frequencies using the uniform rectangular array. Yilmazer [18] presents 2D unitary MP algorithm to estimate the azimuth and elevation angles of the narrowband signal, the computational complexity is reduced through a unitary martix transformation. However, it is necessary to point out that [14]-[18] are the researches on the estimation of some parameters using matrix pencil algorithm in recent years, but there is no joint estimation of time delay and angle. According to signal characteristics, Ding [19] uses the 1D MP algorithm to obtain time delay parameters, and then calculates angle parameters by the trigonometric geometric relation, thus the relative coordinates of the target are obtained. However, this method is limited by the characteristics of the signal itself, which would result in the serious distortion of the estimated result for non ultra wideband signal. The proposed algorithm in the paper is a joint estimation of time delay and angle for OFDM signal based on previous works. The estimated parameters are different, so the corresponding processing methods are also different.

In view of the sample shortage of single snapshot data, combined with the characteristics of OFDM signal, the response of channel frequency domain with time delay and angle parameter information is reconstructed, and the mathematical model suitable for matrix pencil algorithm is established. Through the enhanced two-dimensional matrix pencil algorithm, the virtual system bandwidth and the virtual array aperture are extended. The high precision joint estimation of time delay and angle of arrival under the single snapshot condition can be achieved, and it also has good estimation performance under low SNR. The computation speed is fast by the closed-form solution without pseudo spectral peak searching. In addition, another advantage of the approach is to reduce the overhead of the system. The computational complexity analysis, CRB derivation and simulation are also presented. The simulation results show that the proposed algorithm is an effective method to estimate the location parameters for OFDM signal.

The rest of the paper is organized as follows. Section II introduces the signal model. Section III presents the method of joint TOA and AOA estimation in detail. The computational complexity is compared in Section IV. The CRB for the method is derived in Section V. Simulation and experimental results are presented in Section VI. Finally, conclusions are drawn in Section VII.

Notation: we use the following notation.

T Matrix transpose.

* Matrix conjugate.

н Matrix conjugate transpose.

$E[\bullet]$ Statistical expectation. 
$\otimes \quad$ Kronecker product.

$\odot \quad$ Khatri-Rao product.

\section{Signal Model}

Considering a node equipped with an equidistant line array composed of $M$ antenna elements, as shown in Fig. 1, assuming the distance between the signal source and the antenna array is far enough, $\theta_{i}$ and $\tau_{i}$ represent AOA and propagation delay of the $i$-th multipath signal respectively.

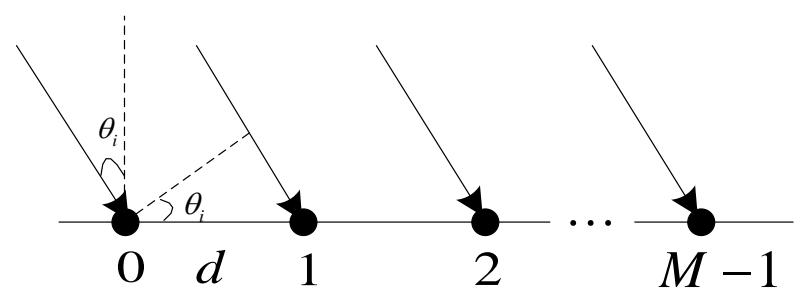

Fig. 1. Uniform antenna array.

$s(t)$ is the OFDM symbol transmitted with $N$ subcarriers, on account of the wireless multipath propagation, the received signal at the $m$-th antenna element can be formulated as,

$$
r_{m}(t)=\sum_{i=0}^{L_{p}-1} a_{i} S\left(t-\tau_{i, m}\right)+n_{m}(t)
$$

where $L_{P}$ is the number of multipath components, and $a_{i}=\left|a_{i}\right| e^{j \varphi_{i}}$ and $\tau_{i, m}$ denote the complex attenuation of the $i$-th path and propagation delay of the $i$-th path in $m$-th antenna respectively. The phase of the complex attenuation $\varphi_{i}$ is normally assumed random from one snapshot to another with a uniform probability density function $U(0,2 \pi)[6], n_{m}(t)$ is additive Gauss white noise. The time delay in each antenna element depends not only on the propagation delay but on the direction of arrival. In particular, for a uniform linear array, the propagation delay associated to the $i$-th arriving path in $m$-th antenna is given by, $\tau_{i, m}=\tau_{i}+\frac{m d \sin \theta_{i}}{c}, \quad 0 \leq m \leq M-1$, with $d$ being the distance between adjacent array elements in the array, $c$ is the speed of light. Taking the Fourier transform of the received signal $r_{m}(t)$ and sampling at $N$ equally spaced frequencies, thus, the discrete frequency-domain channel response of the $k$-th sampling at the $m$-th antenna element is given by

$$
x_{m, k}=H_{m, k}+n_{m, k}=\sum_{i=0}^{L_{p}-1} \alpha_{i} e^{-j 2 \pi f_{c} \tau_{i}} e^{-j 2 \pi \frac{k}{T}\left(\tau_{i}+\frac{m d \sin \theta_{i}}{c}\right)} e^{-j 2 \pi f_{c} \frac{m d \sin \theta_{i}}{c}}+n_{m, k}
$$

where $0 \leq k \leq N-1, f_{c}$ is the carrier frequency, $T=N T_{s}$ is the net data length of OFDM symbol, $T_{S}$ is the sampling interval. It is clear that formula (2) includes the parameter information of TOA and AOA, so the joint estimation method of 2D parameters is discussed as follows. 


\section{Joint TOA and AOA Estimation in OFDM System}

From the above conditions, it is known that the frequency dimension formed by the equal space sampling and the spatial dimension formed by the equidistance antenna array can be considered as an uncorrelated 2D rectangular array, where the frequency dimension contains the TOA information, the spatial dimension contains the AOA information. Therefore, a joint estimation of time delay and angle parameters can be carried out by 2D MP algorithm, the following is the concrete solution process of this method.

\section{A. Information Reconstruction of Channel Frequency Domain}

Because the effect of time delay $\frac{m d \sin \theta_{i}}{c}$ coming from the antenna array is negligible compared with $\tau_{i}$, the upper formula (2) containing TOA and AOA information can be further reconfigured as

$$
x_{m, k}=H_{m, k}+n_{m, k}=\sum_{i=0}^{L_{p}-1} \alpha_{i}^{\prime} y_{i}^{m} z_{i}^{k}+n_{m, k}
$$

where $\alpha_{i}^{\prime}=\alpha_{i} e^{-j 2 \pi f_{c} \tau_{i}}, y_{i}=e^{-j 2 \pi \frac{d \sin \theta_{i}}{\lambda}}, z_{i}=e^{-j 2 \pi \frac{1}{T} \tau_{i}}, n_{m, k}$ denotes additive Gauss white noise with mean zero and variance $\sigma^{2}$ at the $k$-th subcarrier and the $m$-th antenna element. So the vector matrix of the channel frequency-domain reconstruction of the $M$ extended array elements can be expressed as

$$
\boldsymbol{X}=\boldsymbol{H}+\boldsymbol{n}=\boldsymbol{Y}(\boldsymbol{\theta}) \operatorname{diag}[\boldsymbol{\alpha}] \mathbf{Z}^{T}(\boldsymbol{\tau})+\boldsymbol{n}
$$

where $\boldsymbol{\alpha}=\left[\alpha_{0}^{\prime}, \alpha_{1}^{\prime}, \cdots, \alpha_{L_{p}-1}^{\prime}\right]^{T}, \quad \boldsymbol{n} \in \mathbb{C}^{M \times N}$ represents the additive noise, the steering matrix $\boldsymbol{Y}(\boldsymbol{\theta})$ is determined from the antenna geometry as follows

$$
\boldsymbol{Y}(\boldsymbol{\theta})=\left[\boldsymbol{y}\left(\theta_{0}\right), \boldsymbol{y}\left(\theta_{1}\right), \cdots, \boldsymbol{y}\left(\theta_{L_{p}-1}\right)\right]_{M \times L_{p}}
$$

where the steering vector is defined as $y\left(\theta_{i}\right)=\left[1, y_{i}, \cdots, y_{i}^{M-1}\right]^{T}$. While the steering matrix $\mathbf{Z}(\boldsymbol{\tau})$ is determined from the equal space sampling as follows

$$
\mathbf{Z}(\boldsymbol{\tau})=\left[\mathbf{z}\left(\tau_{0}\right), \mathbf{z}\left(\tau_{1}\right), \cdots, \mathbf{z}\left(\tau_{L_{p}-1}\right)\right]_{N \times L_{p}}
$$

where the steering vector is defined as $z\left(\tau_{i}\right)=\left[1, z_{i}, \cdots, z_{i}^{N-1}\right]^{T}$. At the same time, the data matrix of the extended spatial 2D channel frequency response is obtained as

$$
\boldsymbol{X}=\left[\begin{array}{cccc}
x_{0,0} & x_{0,1} & \cdots & x_{0, N-1} \\
x_{1,0} & x_{1,1} & \cdots & x_{1, N-1} \\
\vdots & \vdots & \ddots & \vdots \\
x_{M-1,0} & x_{M-1,1} & \cdots & x_{M-1, N-1}
\end{array}\right]_{M \times N}
$$

From the matrix vector relation of formula (4), we know that the rank of $\boldsymbol{X}$ is no larger than $L_{p}$, i.e., $\operatorname{rank}(\boldsymbol{X}) \leq L_{p}$, which results in that $y_{i}$ and $z_{i}$ cannot be both obtained from the principal left or right singular vectors of $\boldsymbol{X}$ like the 1D MP algorithm. So an enhanced matrix can be defined as 


$$
\boldsymbol{X}_{e}=\left[\begin{array}{cccc}
X_{0} & X_{1} & \cdots & X_{M-K} \\
X_{1} & X_{2} & \cdots & X_{M-K+1} \\
\vdots & \vdots & \ddots & \vdots \\
X_{K-1} & X_{K} & \cdots & X_{M-1}
\end{array}\right]_{K P \times(M-K+1)(N-P+1)}
$$

where

$$
\boldsymbol{X}_{m}=\left[\begin{array}{cccc}
x_{m, 0} & x_{m, 1} & \cdots & x_{m, N-P} \\
x_{m, 1} & x_{m, 2} & \cdots & x_{m, N-P+1} \\
\vdots & \vdots & \ddots & \vdots \\
x_{m, P-1} & x_{m, P} & \cdots & x_{m, N-1}
\end{array}\right]_{P \times(N-P+1)}
$$

So we can attain $\operatorname{rank}\left(\boldsymbol{X}_{e}\right)=L_{p} \geq \operatorname{rank}(\boldsymbol{X}), \quad \boldsymbol{X}_{e}$ is a $K \times(M-K+1)$ Hankel block matrix, and $\boldsymbol{X}_{m}$ is a $P \times(N-P+1)$ Hankel matrix, $K$ and $P$ are the two window parameters, which can be adjusted to increase the estimation accuracy. For the sake of higher reliability and estimation accuracy, some necessary conditions investigated in [17] should be satisfied as

$$
\left\{\begin{array}{l}
M-L_{p}+1 \geq K \geq L_{p}+1 \\
N-L_{p}+1 \geq P \geq L_{p}+1
\end{array}\right.
$$

Using (2) in (9), Hankel matrix $\boldsymbol{X}_{m}$ becomes

$$
\boldsymbol{X}_{m}=\boldsymbol{H}_{m}+\boldsymbol{n}_{m}=\boldsymbol{Z}_{P}(\boldsymbol{\tau}) \operatorname{diag}[\boldsymbol{\alpha}] \mathbf{Y}_{d}^{m}(\boldsymbol{\theta}) \mathbf{Z}_{R}^{T}(\boldsymbol{\tau})+\boldsymbol{n}_{m}
$$

where $\boldsymbol{\alpha}=\left[\alpha_{0}^{\prime}, \alpha_{1}^{\prime}, \cdots, \alpha_{L_{p}-1}^{\prime}\right]^{T}, \mathbf{Z}_{P}(\boldsymbol{\tau})=\left[\mathbf{z}_{P}\left(\tau_{0}\right), \mathbf{z}_{P}\left(\tau_{1}\right), \cdots, \mathbf{z}_{P}\left(\tau_{L_{p}-1}\right)\right]_{P \times L_{p}}, \mathbf{z}_{P}\left(\tau_{i}\right)=\left[1, z_{i}, \cdots, z_{i}^{P-1}\right]^{T}$; $\mathbf{Z}_{R}(\boldsymbol{\tau})=\left[\mathbf{z}_{R}\left(\tau_{0}\right), \mathbf{z}_{R}\left(\tau_{1}\right), \cdots, \mathbf{z}_{R}\left(\tau_{L_{p}-1}\right)\right]_{(N-P-1) \times L_{p}} \quad, \quad \mathbf{z}_{R}\left(\tau_{i}\right)=\left[1, z_{i}, \cdots, z_{i}^{N-P}\right]^{T} \quad ;$ $\boldsymbol{Y}_{d}(\boldsymbol{\theta})=\operatorname{diag}\left(y_{0}, y_{1}, \cdots, y_{L_{p}-1}\right)$. Then, using (11) in (8), $\boldsymbol{X}_{e}$ becomes

$$
\boldsymbol{X}_{e}=\boldsymbol{E}_{P} \operatorname{diag}[\boldsymbol{\alpha}] \boldsymbol{E}_{R}^{T}+\boldsymbol{n}_{e}
$$

where

$$
\boldsymbol{E}_{P}=\left[\begin{array}{l}
\boldsymbol{Z}_{P}(\boldsymbol{\tau}) \\
\boldsymbol{Z}_{P}(\boldsymbol{\tau}) \boldsymbol{Y}_{d}(\boldsymbol{\theta}) \\
\cdots \\
\boldsymbol{Z}_{P}(\boldsymbol{\tau}) \boldsymbol{Y}_{d}^{K-1}(\boldsymbol{\theta})
\end{array}\right], \quad \boldsymbol{E}_{R}=\left[\boldsymbol{Z}_{R}^{T}(\boldsymbol{\tau}), \boldsymbol{Y}_{d}(\boldsymbol{\theta}) \mathbf{Z}_{R}^{T}(\boldsymbol{\tau}), \cdots, \boldsymbol{Y}_{d}^{M-K}(\boldsymbol{\theta}) \mathbf{Z}_{R}^{T}(\boldsymbol{\tau})\right] .
$$

\section{B. AOA Estimation}

The singular value decomposition (SVD) of $\boldsymbol{X}_{e}$ has the form

$$
\boldsymbol{X}_{e}=\sum_{i=0}^{J-1} \sigma_{i} u_{i} v_{i}^{H}=\boldsymbol{U}_{s} \sum_{s} \boldsymbol{V}_{s}^{H}+\boldsymbol{U}_{n} \sum_{n} \boldsymbol{V}_{n}^{H},
$$

where $J=\min (K P,(M-K+1)(N-P+1)), \sigma_{0} \geq \sigma_{1} \geq \cdots \geq \sigma_{J-1}$, without loss of generality, $\boldsymbol{U}_{s}=\left[u_{0}, u_{1}, \cdots, u_{L_{p}-1}\right], \sum_{s}=\operatorname{diag}\left[\sigma_{1}, \sigma_{2}, \cdots, \sigma_{L p}\right]$ and $\boldsymbol{V}_{s}=\left[v_{1}, v_{2}, \cdots, v_{L_{p}}\right]$ are in the signal subspace corresponding to the $L_{p}$ principal components whereas $\boldsymbol{U}_{n}=\left[u_{L_{P}+1}, u_{L_{P}+2}, \cdots, u_{J}\right]$, $\sum_{n}=\operatorname{diag}\left[\sigma_{L_{p}+1}, \sigma_{L_{p}+2}, \cdots, \sigma_{J}\right]$, and $\boldsymbol{V}_{n}=\left[v_{L_{p}+1}, v_{L_{p}+2}, \cdots, v_{J}\right]$ are in the noise subspace.

Since $\boldsymbol{E}_{P}$ and $\boldsymbol{U}_{s}$ have the same signal sub-space, there is only one $L_{p} \times L_{p}$ nonsingular 
matrix $\boldsymbol{T}_{1}$, which satisfies $\boldsymbol{E}_{P} \times \boldsymbol{T}_{1}=\boldsymbol{U}_{s}$. Along the spatial dimension, the matrix pencil can be written as $\boldsymbol{U}_{s 2}-y \boldsymbol{U}_{s 1}$, where $\boldsymbol{U}_{s 1}$ and $\boldsymbol{U}_{s 2}$ are gained from $\boldsymbol{U}_{s}$ with the last $P$ lows deleted and the first $P$ lows deleted respectively. So the expected parameter $\hat{y}_{i}$ can be estimated from the eigenvalues of the matrix $\boldsymbol{U}_{s 1}^{\dagger} \boldsymbol{U}_{s 2}$ [14], where $\boldsymbol{U}_{s 1}^{\dagger}$ is the Moore-Penrose pseudo-inverse of $\boldsymbol{U}_{s 1}$, according to $\hat{y}_{i}=e^{-j 2 \pi \frac{d \sin \theta_{i}}{\lambda}}$, the AOA of the $i$-th multipath can be calculated as

$$
\hat{\theta}_{i}=\arcsin \left[-\operatorname{angle}\left(\hat{y}_{i}\right) \lambda /(2 \pi d)\right], \quad i=0,1, \cdots, L_{P}-1 \text {. }
$$

\section{TOA Estimation}

In order to extract the other set of poles $\left\{\hat{z}_{i} ; i=0,1, \cdots, L_{P}-1\right\}$, the shuffling matrix is used to introduce the permutation as, $\boldsymbol{U}_{s p}=\boldsymbol{C} \boldsymbol{U}_{s}$, where the shuffling matrix $\boldsymbol{C}$ is defined as follows

$$
\begin{aligned}
\boldsymbol{C}= & {[c(1), c(1+P), \cdots, c(1+(K-1) P), c(2), c(2+P), \cdots,} \\
& c(2+(K-1) P), \cdots, c(P), c(P+P), \cdots, c(P+(K-1) P)]^{T}
\end{aligned}
$$

where $c(i)$ is a $K P \times 1$ vector with one at the $i$-th position and zero everywhere else. Along the frequency dimension, the matrix pencil can be written as $\boldsymbol{U}_{s p 2}-z \boldsymbol{U}_{s p 1}$, where $\boldsymbol{U}_{s p 1}$ and $\boldsymbol{U}_{\text {sp2 }}$ are gained from $\boldsymbol{U}_{s p}$ with the last $K$ lows deleted and the first $K$ lows deleted respectively. So the expected parameter $\hat{z}_{i}$ can be estimated from the eigenvalues of the matrix $\boldsymbol{U}_{s p 1}^{\dagger} \boldsymbol{U}_{s p 2}$, where $\boldsymbol{U}_{s p 1}^{\dagger}$ is the Moore-Penrose pseudo-inverse of $\boldsymbol{U}_{s p 1}$, according to $\hat{z}_{i}=e^{-j 2 \pi \frac{1}{T} \tau_{i}}$, the TOA of the $i$-th multipath can be calculated as

$$
\hat{\tau}_{i}=-\operatorname{angle}\left(\hat{z}_{i}\right) T / 2 \pi, \quad i=0,1, \cdots, L_{P}-1 .
$$

\section{Parameter Pairing}

The order of poles in each set above, namely $\left\{\hat{\theta}_{i} ; i=0,1, \cdots, L_{P}-1\right\}$ and $\left\{\hat{\tau}_{i} ; i=0,1, \cdots, L_{P}-1\right\}$, is still unknown. That is, the $\hat{\theta}_{i}$ in $\left\{\hat{\theta}_{i} ; i=0,1, \cdots, L_{P}-1\right\}$ is not necessarily the $\hat{\theta}_{i}$ in the correct pairs $\left\{\left(\hat{\theta}_{i}, \hat{\tau}_{i}\right) ; i=0,1, \cdots, L_{P}-1\right\}$. To obtain the correct pairing, we need to pair the two sets $\left\{\hat{y}_{i} ; i=0,1, \cdots, L_{P}-1\right\}$ and $\left\{\hat{z}_{i} ; i=0,1, \cdots, L_{P}-1\right\}$, We first perform the eigenvalue decomposition of $\boldsymbol{U}_{s 1}^{\dagger} \boldsymbol{U}_{s 2}$ and $\boldsymbol{U}_{s p 1}^{\dagger} \boldsymbol{U}_{s p 2}$ as follows

$$
\left\{\begin{array}{l}
\boldsymbol{U}_{s 1}^{\dagger} \boldsymbol{U}_{s 2}=\boldsymbol{T}_{a}^{-1} \boldsymbol{F}_{a} \boldsymbol{T}_{a} \\
\boldsymbol{U}_{s p 1}^{\dagger} \boldsymbol{U}_{s p 2}=\boldsymbol{T}_{b}^{-1} \boldsymbol{G}_{b} \boldsymbol{T}_{b}
\end{array}\right.
$$

then the eigenvalue decomposition of a linear combination of $\boldsymbol{U}_{s 1}^{\dagger} \boldsymbol{U}_{s 2}$ and $\boldsymbol{U}_{s p 1}^{\dagger} \boldsymbol{U}_{s p 2}$ is computed as $\beta \boldsymbol{U}_{s 1}^{\dagger} \boldsymbol{U}_{s 2}+(1-\beta) \boldsymbol{U}_{s p 1}^{\dagger} \boldsymbol{U}_{s p 2}=\boldsymbol{T}^{-1} \boldsymbol{D} \boldsymbol{T}$, where the parameter $\beta$ is scalar. According to [20], the permutation matrices $\boldsymbol{Q}_{1}=\boldsymbol{T} \boldsymbol{T}_{a}^{-1}$ and $\boldsymbol{Q}_{2}=\boldsymbol{T T}_{b}^{-1}$ are constructed, then the positions of the maximum element of each row of $\boldsymbol{Q}_{1}$ and $\boldsymbol{Q}_{2}$ are put in two vectors $q_{a}$ and $q_{b}$. Finally, the elements $\hat{y}_{i}$ of $\boldsymbol{F}_{a}$ are classified using the positions given by $q_{a}$; the elements $\hat{z}_{i}$ of $\boldsymbol{G}_{b}$ are classified using the positions given by $q_{b}$. Here, we can get the correct pairing of 
$\left\{\left(\hat{\theta}_{i}, \hat{\tau}_{i}\right) ; i=0,1, \cdots, L_{P}-1\right\}$. In conclusion, the pairing algorithm does not require any search procedure compared with that pairing operation in [17].

\section{E. Algorithm steps}

To further review the method, Fig. 2 shows the functional block diagram of the proposed algorithm.

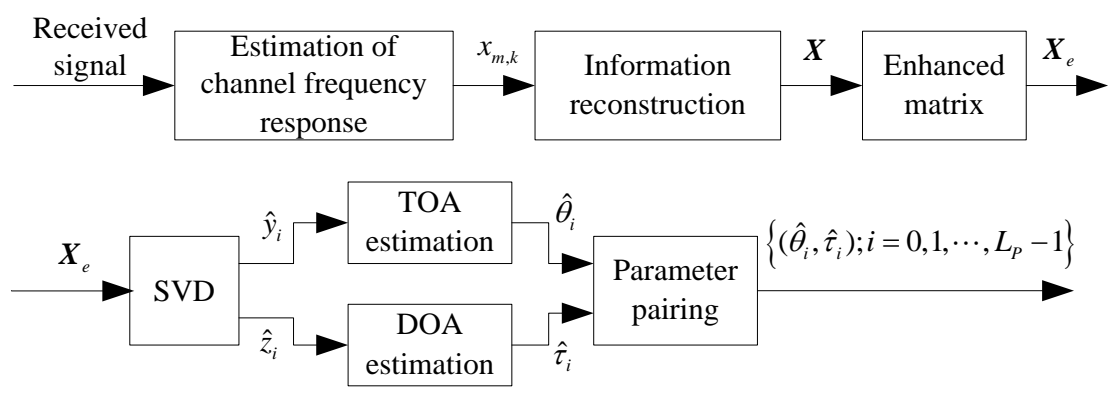

Fig. 2. Functional block diagram of the proposed algorithm.

Step 1: the received signal is first used to estimated channel frequency response.

Step 2: use the formula (7) to get the data matrix of the channel frequency-domain reconstruction of the $M$ extended array elements, $\boldsymbol{X}$.

Step 3: according to the formula (8) to get the enhanced matrix $\boldsymbol{X}_{e}$.

Step 4: $\boldsymbol{U}_{s}$ and $\boldsymbol{U}_{s 1}$ are obtained by singular value decomposition of the formula (13), thus the expected parameter $\hat{y}_{i}$ can be estimated from the eigenvalues of the matrix $\boldsymbol{U}_{s 1}^{\dagger} \boldsymbol{U}_{s 2}$.

Step 5: introduce the permutation as, $\boldsymbol{U}_{s p}=\boldsymbol{C} \boldsymbol{U}_{s}$, then the expected parameter $\hat{z}_{i}$ can be estimated from the eigenvalues of the matrix $\boldsymbol{U}_{s p 1}^{\dagger} \boldsymbol{U}_{s p 2}$.

Step 6: according to $\hat{y}_{i}=e^{-j 2 \pi \frac{d \sin \theta_{i}}{\lambda}}, \hat{\theta}_{i}$ can be calculated; Similarly, according to $\hat{z}_{i}=e^{-j 2 \pi \frac{1}{T} \tau_{i}}$, $\hat{\tau}_{i}$ can be obtained.

Step 7: finally, the pairing of $\hat{\theta}_{i}$ and $\hat{\tau}_{i}$ is completed according to the pairing method, i.e., $\left\{\left(\hat{\theta}_{i}, \hat{\tau}_{i}\right) ; i=0,1, \cdots, L_{P}-1\right\}$.

\section{Computational Complexity Analysis}

The computational complexity of the algorithm in this paper mainly consists of the following two aspects: the SVD of the enhanced matrix $\boldsymbol{X}_{e}$, the computational complexity is $O\left(17 K^{3} P^{3} / 3+K^{2} P^{2}(M-K+1)(N-P+1)\right)$; the complexity of $\boldsymbol{U}_{s 2}-y \boldsymbol{U}_{s 1}$ and $\boldsymbol{U}_{s p 2}-z \boldsymbol{U}_{s p 1}$ is $O\left(3 L_{p}^{2} K P\right)$. So the total complexity of the algorithm is $O\left(17 K^{3} P^{3} / 3+K^{2} P^{2}(M-K+1)(N-P+1)+3 L_{p}^{2} K P\right)$. The computational complexity of using Root-MUSIC algorithm in [11] mainly consists of three aspects: the complexity of covariance matrix calculation and eigen-decomposition is $O\left(M^{3}+N^{3}+M^{2}+N^{2}\right)$; the complexity of 
constructing roots polynomial is $O\left(M^{2}-M L_{p}+N^{2}-N L_{p}\right)$; the complexity of the parameter pairing is $O\left((2 N)^{2}+(2 N)^{3}+(2 N)^{2} L_{p} !+4 N L_{p} !-2 N L_{p} L_{p} !\right)$. The complexity of $1 \mathrm{D}$ MP algorithm adopted in [16] and [19] is $O\left(3 M^{2}+3 N^{2}+6 M L_{p}^{2}+6 N L_{p}^{2}+2 M+2 N-4 L_{p}^{3}\right)$, Table 1 compares the computational complexity of the three algorithms.

Table 1. Algorithm complexity comparison

\begin{tabular}{cc}
\hline Algorithm type & Computational complexity \\
\hline Proposed algorithm & $O\left(17 K^{3} P^{3} / 3+K^{2} P^{2}(M-K+1)(N-P+1)+3 L_{p}^{2} K P\right)$ \\
Root-MUSIC & $O\left(M^{3}+9 N^{3}+2 M^{2}+N^{2}\left(6+4 L_{p} !\right)-L_{p}(M+N)+\left(4-2 L_{p}\right) N L_{p} !\right)$ \\
1D MP & $O\left(3 M^{2}+3 N^{2}+6 M L_{p}^{2}+6 N L_{p}^{2}+2 M+2 N-4 L_{p}^{3}\right)$ \\
\hline
\end{tabular}

It can be seen from Table 1 that the complexity of the Root-MUSIC algorithm increases exponentially with the number of elements $M$ and samplings $N$. However, the complexity of the proposed algorithm is mainly related to the window adjustment parameters $K$ and $P$ of the enhanced matrix, and is slightly higher than that of 1D MP algorithm.

\section{The Cramer-Rao Bound}

The CRB is the lower bound of minimum variance which an unbiased estimation can achieve. The following is to derive the CRB of the model. By using the general relation $\operatorname{vec}[\boldsymbol{A} \operatorname{diag}(\mathrm{b}) \boldsymbol{C}]=\left(\boldsymbol{C}^{T} \odot \boldsymbol{A}\right) \mathrm{b}$ in [21], the vector operation of the matrix $\boldsymbol{H}$ in (4) can be obtained

$$
\operatorname{vec}[\boldsymbol{H}]=[\boldsymbol{Z}(\boldsymbol{\tau}) \odot \boldsymbol{Y}(\boldsymbol{\theta})] \boldsymbol{\alpha}=\boldsymbol{U}(\boldsymbol{\theta}, \boldsymbol{\tau}) \boldsymbol{\alpha}
$$

where $\odot$ denotes Khatri-Rao product, $\boldsymbol{U}(\boldsymbol{\theta}, \boldsymbol{\tau}) \in \mathbb{C}^{M N \times L_{p}}$ is the space-frequency response matrix with $L_{p}$ paths and is defined as

$$
\boldsymbol{U}(\boldsymbol{\theta}, \boldsymbol{\tau})=\boldsymbol{Z}(\boldsymbol{\tau}) \odot \boldsymbol{Y}(\boldsymbol{\theta})=\left[\boldsymbol{u}\left(\theta_{0}, \tau_{0}\right), \cdots, \boldsymbol{u}\left(\theta_{L_{p}-1}, \tau_{L_{p}-1}\right)\right]
$$

where $\boldsymbol{u}\left(\theta_{i}, \tau_{i}\right)=\mathbf{z}\left(\tau_{i}\right) \otimes \boldsymbol{y}\left(\theta_{i}\right), \otimes$ represents Kronecker product. The general form of (18) including noise is then given by

$$
\boldsymbol{g}=\operatorname{vec}[\hat{\boldsymbol{X}}]=\boldsymbol{U}(\boldsymbol{\theta}, \boldsymbol{\tau}) \boldsymbol{\alpha}+\boldsymbol{w}
$$

where $\boldsymbol{w}=\operatorname{vec}[\boldsymbol{n}]$ denotes a complex, stationary, zero-mean Gaussian random process that is uncorrelated for the different paths. So we can obtain $E\left[\boldsymbol{w} \boldsymbol{w}^{H}\right]=\sigma_{h}^{2} \boldsymbol{I}$ and $E\left[\boldsymbol{w} \boldsymbol{w}^{T}\right]=0$, where $\sigma_{h}^{2}$ is the variance of the entries of the estimation noise matrix $\boldsymbol{n}$.

Define the parameter vector as $\Theta=\left[\begin{array}{ll}\sigma^{2} & \eta^{T}\end{array}\right]^{T}, \boldsymbol{\eta}=\left[\begin{array}{ll}\boldsymbol{\theta}^{T} & \boldsymbol{\tau}^{T}\end{array}\right]^{T}$. According to (20) and the relevant initial conditions, the likelihood probability density function of $\boldsymbol{g}$ is

$$
f(\boldsymbol{g} / \Theta, \boldsymbol{\alpha})=\prod_{j=1}^{K} \frac{1}{(2 \pi)^{M N}\left(\sigma_{h}^{2} / 2\right)^{M N}} \exp \left(-\frac{1}{\sigma_{h}^{2}}\|\boldsymbol{g}-\boldsymbol{U} \boldsymbol{\alpha}\|_{2}^{2}\right)
$$

Thus, the log-likelihood function of (21), with omitting the constant term, is 


$$
\ln f=-M N K \ln \sigma_{h}^{2}-\frac{1}{\sigma_{h}^{2}}[\boldsymbol{g}-\boldsymbol{U} \boldsymbol{\alpha}]^{\mathrm{H}}[\boldsymbol{g}-\boldsymbol{U} \boldsymbol{\alpha}]
$$

To calculate the Fisher Information Matrix (FIM), the derivatives of (22) with respect to $\sigma^{2}$, the real part $\operatorname{Re} \boldsymbol{\alpha}$ of $\boldsymbol{\alpha}$, and the imaginary part $\operatorname{Im} \boldsymbol{\alpha}$ of $\boldsymbol{\alpha}$ can be obtained as

$$
\begin{gathered}
\frac{\partial \ln f}{\partial\left(\sigma_{h}^{2}\right)}=-\frac{M N}{\sigma_{h}^{2}}+\frac{1}{\sigma_{h}^{4}} \boldsymbol{w}^{\mathrm{H}} \boldsymbol{w}, \quad \frac{\partial \ln f}{\partial \operatorname{Re} \boldsymbol{\alpha}}=\frac{2}{\sigma_{h}^{2}} \operatorname{Re}\left[\boldsymbol{U}^{\mathrm{H}} \boldsymbol{w}\right], \\
\frac{\partial \ln f}{\partial \operatorname{Im} \boldsymbol{\alpha}}=\frac{2}{\sigma_{h}^{2}} \operatorname{Im}\left[\boldsymbol{U}^{\mathrm{H}} \boldsymbol{w}\right], \quad \frac{\partial \ln f}{\partial \theta_{i}}=\frac{2}{\sigma_{h}^{2}} \operatorname{Re}\left[\alpha_{i}^{\prime} \boldsymbol{d}_{\theta_{i}}^{\mathrm{H}} \boldsymbol{w}\right], \\
\frac{\partial \ln f}{\partial \tau_{i}}=\frac{2}{\sigma_{h}^{2}} \operatorname{Re}\left[\alpha_{i}^{\prime} \boldsymbol{d}_{\tau_{i}}^{\mathrm{H}} \boldsymbol{w}\right], \quad i=0, \cdots, L_{P}-1,
\end{gathered}
$$

where $\boldsymbol{d}_{\theta_{i}}$ is the derivative with respect to $\theta_{i}$ of the $i$-th of $\boldsymbol{U}, \boldsymbol{d}_{\tau_{i}}$ is the derivative with respect to $\tau_{i}$ of the $i$-th of $\boldsymbol{U}$, namely, $\boldsymbol{d}_{\theta_{i}}=\mathbf{z}\left(\tau_{i}\right) \otimes \partial \boldsymbol{y}\left(\theta_{i}\right) / \partial \theta_{i}, \boldsymbol{d}_{\tau_{i}}=\partial \mathbf{z}\left(\tau_{i}\right) / \partial \tau_{i} \otimes \boldsymbol{y}\left(\theta_{i}\right)$. Further, written more compactly

$$
\begin{aligned}
& \frac{\partial \ln f}{\partial \boldsymbol{\theta}}=\frac{2}{\sigma_{h}^{2}} \operatorname{Re}\left[(\operatorname{diag}[\boldsymbol{\alpha}])^{\mathrm{H}} \boldsymbol{D}_{\boldsymbol{\theta}}^{\mathrm{H}} \boldsymbol{w}\right] \\
& \frac{\partial \ln f}{\partial \boldsymbol{\tau}}=\frac{2}{\sigma_{h}^{2}} \operatorname{Re}\left[(\operatorname{diag}[\boldsymbol{\alpha}])^{\mathrm{H}} \boldsymbol{D}_{\tau}^{\mathrm{H}} \boldsymbol{w}\right]
\end{aligned}
$$

where $\boldsymbol{D}_{\boldsymbol{\theta}}=\partial \boldsymbol{U} / \partial \boldsymbol{\theta}=\boldsymbol{Z} \odot \boldsymbol{Y}^{\prime}=\left[\boldsymbol{d}_{\theta_{0}}, \cdots, \boldsymbol{d}_{\theta_{L p-1}}\right], \quad \boldsymbol{D}_{\boldsymbol{\tau}}=\partial \boldsymbol{U} / \partial \boldsymbol{\tau}=\boldsymbol{Z}^{\prime} \odot \boldsymbol{Y}=\left[\boldsymbol{d}_{\tau_{0}}, \cdots, \boldsymbol{d}_{\tau_{L_{p-1}}}\right]$. So the derivative of $\ln f$ with respect to the parameter $\boldsymbol{\eta}=\left[\begin{array}{ll}\boldsymbol{\theta}^{T} & \boldsymbol{\tau}^{T}\end{array}\right]^{T}$ can be derived as

$$
\frac{\partial \ln f}{\partial \boldsymbol{\eta}}=\frac{2}{\sigma_{h}^{2}} \operatorname{Re}\left[\alpha_{0}^{\prime} \boldsymbol{d}_{\theta_{0}}^{\mathrm{H}} \boldsymbol{w}, \cdots, \alpha_{L_{p}-1}^{\prime} \boldsymbol{d}_{{t_{p}-1}_{1}}^{\mathrm{H}} \boldsymbol{w}, \alpha_{0}^{\prime} \boldsymbol{d}_{\tau_{0}}^{\mathrm{H}} \boldsymbol{w}, \cdots, \alpha_{L_{p}-1}^{\prime} \boldsymbol{d}_{\tau_{L_{p}-1}}^{\mathrm{H}} \boldsymbol{w}\right]
$$

Let $\boldsymbol{D}=\left[\begin{array}{ll}\boldsymbol{D}_{\tau} & \boldsymbol{D}_{\boldsymbol{\theta}}\end{array}\right]_{M N \times 2 L_{P}}$, and $\boldsymbol{B}=\boldsymbol{I}_{2} \otimes \operatorname{diag}[\boldsymbol{\alpha}]$, then (25) can be further simplified as

$$
\frac{\partial \ln f}{\partial \boldsymbol{\eta}}=\frac{2}{\sigma_{h}^{2}} \operatorname{Re}\left[\boldsymbol{B}^{H} \boldsymbol{D}^{\mathrm{H}} \boldsymbol{w}\right]
$$

Using results proven in [22], we first note that $\partial \ln f / \partial\left(\sigma_{h}^{2}\right)$ is not correlated with any of the other derivatives computed above. Then, the following expressions can be attained as

$$
\begin{gathered}
E\left[\left[\frac{\partial \ln f}{\partial\left(\sigma_{h}^{2}\right)}\right]^{2}\right]=\frac{M N}{\sigma_{h}^{4}} \\
E\left[\left[\frac{\partial \ln f}{\partial \operatorname{Re} \boldsymbol{\alpha}}\right]\left[\frac{\partial \ln f}{\partial \operatorname{Re} \boldsymbol{\alpha}}\right]^{\mathrm{T}}\right]=\frac{2}{\sigma_{h}^{2}} \operatorname{Re}\left[\boldsymbol{U}^{\mathrm{H}} \boldsymbol{U}\right] \\
E\left[\left[\frac{\partial \ln f}{\partial \operatorname{Re} \boldsymbol{\alpha}}\right]\left[\frac{\partial \ln f}{\partial \operatorname{Im} \boldsymbol{\alpha}}\right]^{\mathrm{T}}\right]=\frac{-2}{\sigma_{h}^{2}} \operatorname{Im}\left[\boldsymbol{U}^{\mathrm{H}} \boldsymbol{U}\right] \\
E\left[\left[\frac{\partial \ln f}{\partial \operatorname{Im} \boldsymbol{\alpha}}\right]\left[\frac{\partial \ln f}{\partial \operatorname{Im})}\right]^{\mathrm{T}}\right]=\frac{2}{\sigma_{h}^{2}} \operatorname{Re}\left[\boldsymbol{U}^{\mathrm{H}} \boldsymbol{U}\right]
\end{gathered}
$$




$$
\begin{gathered}
E\left[\left[\frac{\partial \ln f}{\partial \operatorname{Re} \boldsymbol{\alpha}}\right]\left[\frac{\partial \ln f}{\partial \boldsymbol{\eta}}\right]^{\mathrm{T}}\right]=\frac{2}{\sigma_{h}^{2}} \operatorname{Re}\left[\boldsymbol{U}^{\mathrm{H}} \boldsymbol{D} \boldsymbol{B}\right] \\
E\left[\left[\frac{\partial \ln f}{\partial \operatorname{Im} \boldsymbol{\alpha}}\right]\left[\frac{\partial \ln f}{\partial \boldsymbol{\eta}}\right]^{\mathrm{T}}\right]=\frac{2}{\sigma^{2}} \operatorname{Im}\left[\boldsymbol{U}^{\mathrm{H}} \boldsymbol{D} \boldsymbol{B}\right] \\
E\left[\left[\frac{\partial \ln f}{\partial \boldsymbol{\eta}}\right]\left[\frac{\partial \ln f}{\partial \boldsymbol{\eta}}\right]^{\mathrm{T}}\right]=\frac{2}{\sigma_{h}^{2}} \operatorname{Re}\left[\boldsymbol{B}^{\mathrm{H}} \boldsymbol{D}^{\mathrm{H}} \boldsymbol{D} \boldsymbol{B}\right]
\end{gathered}
$$

The FIM of the deterministic parameters is obtained by $E\left[\left[\frac{\partial \ln f}{\partial[\Theta, \alpha]}\right]\left[\frac{\partial \ln f}{\partial[(\Theta, \alpha)]}\right]^{\mathrm{T}}\right]$, where $\partial \ln f / \partial[(\Theta, \boldsymbol{\alpha})]=\partial \ln f / \partial\left[\sigma_{h}^{2}, \boldsymbol{\eta}^{T},(\operatorname{Re} \boldsymbol{\alpha})^{T},(\operatorname{Im} \boldsymbol{\alpha})^{T}\right]$. Finally, using the results in [22], we can show that the CRB for the parameters of delay and angle is

$$
C R B(\boldsymbol{\eta})=\frac{\sigma_{h}^{2}}{2}\left\{\operatorname{Re}\left[\boldsymbol{B}^{\mathrm{H}} \boldsymbol{D}^{\mathrm{H}}\left[\boldsymbol{I}-\boldsymbol{U} \boldsymbol{U}^{\dagger}\right] \boldsymbol{D} \boldsymbol{B}\right]\right\}^{-1} .
$$

which completes the proof.

\section{Simulation Results}

In this section, the performance of joint TOA and AOA estimation for the single snapshot data is studied. Monte Carlo simulation is utilized to verify the practicability and robustness of the proposed algorithm. The relevant parameters of narrowband array antenna in OFDM system are set as follows, the system bandwidth is $B=20 \mathrm{MHz}$, the FFT cycle is $T=3.2 \mu \mathrm{s}$, the number of subcarriers is $N=64$, the carrier frequency is $f_{c}=2.4 \mathrm{GHz}$, the number of array elements is $M=12$, the array spacing is $d=0.0625$ meters, etc.

Firstly, we define the Root Mean Square Error (RMSE) as

$$
R M S E_{i}=\sqrt{\frac{1}{S} \sum_{s=0}^{S-1}\left(\hat{x}_{s, i}-x_{i}\right)^{2}}
$$

where $S$ is Monte Carlo simulation times, $\hat{x}_{s, i}$ indicates the estimated value of the $i$-th multipath in the $s$-th simulation, $x_{i}$ is the true value of the corresponding $i$-th multipath parameter.

Simulation 1: assuming that the multipath number of the received signals is $L_{P}=3$; the arrival time is $\tau_{0}=100 \mathrm{~ns}, \tau_{1}=140 \mathrm{~ns}, \tau_{2}=180 \mathrm{~ns}$, respectively; the direction of arrival is $\theta_{0}=30^{\circ}, \theta_{1}=40^{\circ}, \theta_{2}=60^{\circ}$, respectively; three corresponding complex multipath fading coefficients are $1,0.9$ and 0.8 . Simulation times of the algorithm is $S=200$, under different SNRs, the TOA and AOA RMSE of the three multipath components are shown in Table 2 and

\section{Table 3.}


Table 2. Relationship between RMSE of TOA and SNR

\begin{tabular}{cccccccc}
\hline $\mathrm{SNR} / \mathrm{dB}$ & 0 & 5 & 10 & 15 & 20 & 25 & 30 \\
\hline$R M S E_{0} / \mathrm{ns}$ & 1.5425 & 0.8258 & 0.4895 & 0.2706 & 0.1475 & 0.0823 & 0.0462 \\
$R M S E_{1} / \mathrm{ns}$ & 2.4053 & 1.1061 & 0.6176 & 0.3670 & 0.1937 & 0.1168 & 0.0667 \\
$R M S E_{2} / \mathrm{ns}$ & 1.7974 & 0.9149 & 0.5557 & 0.2943 & 0.1671 & 0.0983 & 0.0525 \\
\hline
\end{tabular}

Table 3. Relationship between RMSE of AOA and SNR

\begin{tabular}{cccccccc}
\hline $\mathrm{SNR} / \mathrm{dB}$ & 0 & 5 & 10 & 15 & 20 & 25 & 30 \\
\hline $\mathrm{RMSE}_{0} /^{\circ}$ & 0.3000 & 0.1713 & 0.0901 & 0.0529 & 0.0273 & 0.0168 & 0.0093 \\
$\mathrm{RMSE}_{1} /^{\circ}$ & 0.4060 & 0.2341 & 0.1372 & 0.0684 & 0.0399 & 0.0239 & 0.0122 \\
$\mathrm{RMSE}_{2} /^{\circ}$ & 0.5253 & 0.2768 & 0.1376 & 0.0754 & 0.0455 & 0.0283 & 0.0146 \\
\hline
\end{tabular}

It is clear in Table 1 and Table 2 that the RMSE performance of TOA and AOA decreases with the increase of SNR. Namely the higher SNR, the better the joint estimation result. In the case of $\mathrm{SNR}=0 \mathrm{~dB}$, the TOA estimation error of the first path is less than 2ns, the AOA estimation error of the first path is less than 0.5 degree, which can well meet the positioning requirements.

Simulation 2: in the case of $\mathrm{SNR}=0 \mathrm{~dB}$ and $10 \mathrm{~dB}$, other conditions are the same as simulation 1, Simulation times of the algorithm is $S=200$. The scatter diagrams of the joint estimation of TOA and AOA combined with three multipath components are obtained. Fig. 3 and Fig. 4 show that the algorithm has good performance for the joint estimation under the condition of low SNR, and at SNR=10 dB, the distribution of joint estimates is more concentrated, which has higher estimation accuracy.



Fig. 3. Joint TOA and AOA estimation, $\mathrm{SNR}=0 \mathrm{~dB}$.

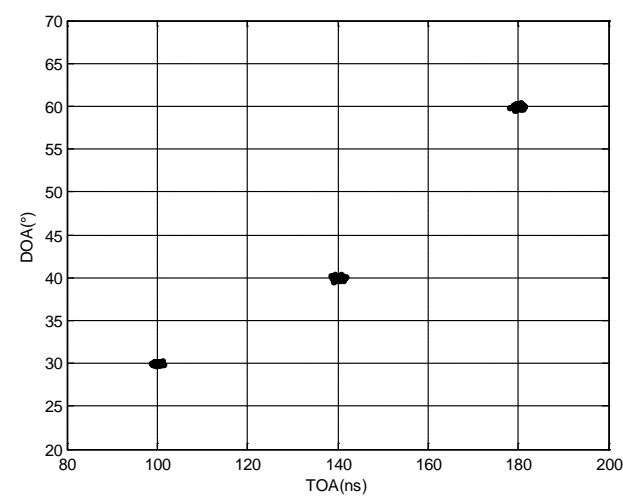

Fig. 4. Joint TOA and AOA estimation, $\mathrm{SNR}=10 \mathrm{~dB}$.

Simulation 3: at low SNR of SNR $=5 \mathrm{~dB}$, other conditions are the same as simulation 1 . In the $S=200$ Monte Carlo simulations, we can obtain the TOA estimation $\hat{\tau}_{s, 1}, \hat{\tau}_{s, 2}, \hat{\tau}_{s, 3}$ of the three multipath components for each simulation, and the corresponding AOA estimation $\hat{\theta}_{s, 1}$, $\hat{\theta}_{s, 2}, \hat{\theta}_{s, 3}$. Those estimation distributions are shown in Fig. 5 and Fig. 6, it can be seen that the 
estimation of TOA and AOA, under the conditions of single snapshot and low SNR, is still close to the true value. So the proposed algorithm has higher practicability.

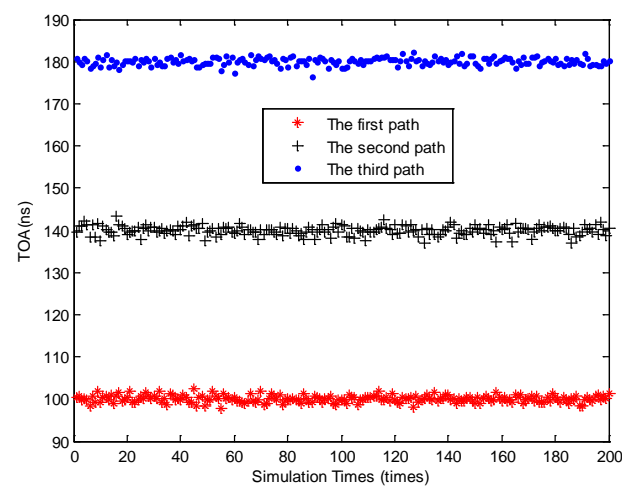

Fig. 5. TOA distribution, $\mathrm{SNR}=5 \mathrm{~dB}$.

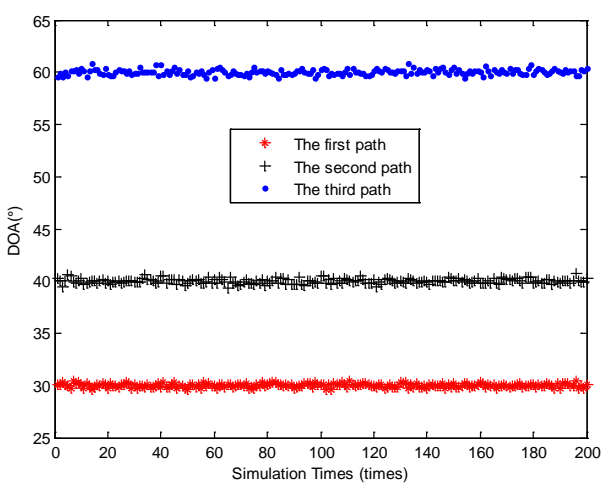

Fig. 6. AOA distribution, $\mathrm{SNR}=5 \mathrm{~dB}$.

The estimation error of TOA and AOA is defined as $\Delta \tau_{s, i}=\hat{\tau}_{s, i}-\tau_{i}$ and $\Delta \theta_{s, i}=\hat{\theta}_{s, i}-\theta_{i}$, respectively. Then the scatter diagram of $\Delta \tau_{s, 1}, \Delta \tau_{s, 2}, \Delta \tau_{s, 3}$ corresponding to three multipath components is shown as Fig. 7, and the corresponding $\Delta \theta_{s, 1}, \Delta \theta_{s, 2}, \Delta \theta_{s, 3}$ distribution, is shown in Fig. 8. It can be seen from Fig. 7 and Fig. 8 that the estimation errors of TOA and AOA, at low SNR, are only occasionally divergent, and generally tend to be stable. Therefore, this proposed algorithm has strong robustness under the condition of low SNR.

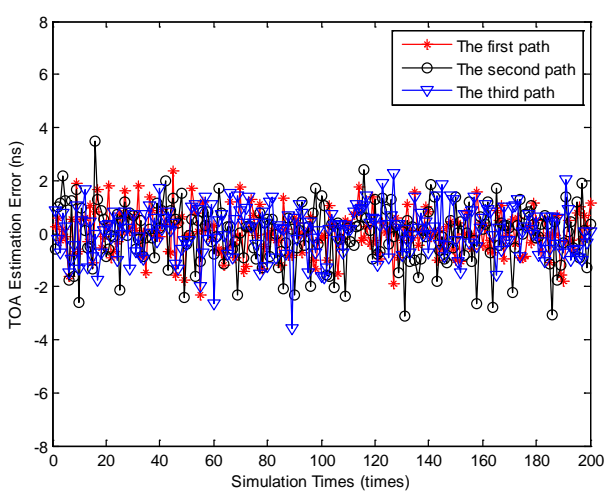

Fig. 7. TOA error distribution, $\mathrm{SNR}=5 \mathrm{~dB}$;

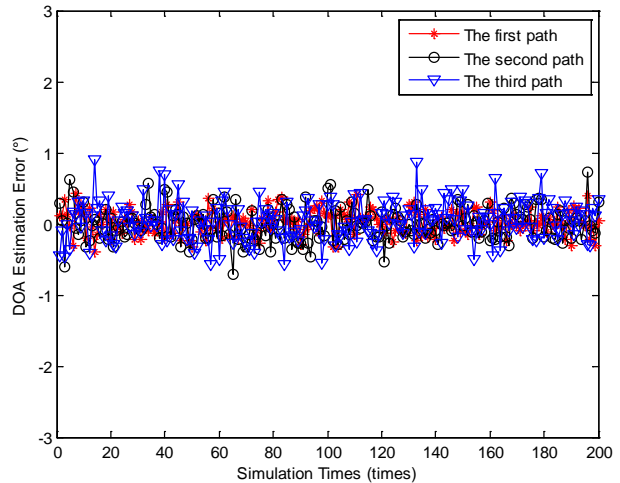

Fig. 8. AOA error distribution, $S N R=5 d B$.

Simulation 4: 200 Monte Carlo simulations are performed on the condition of different multipath numbers, and the TOA and AOA estimation performances of the respective first path are obtained respectively, as shown in Figs. 9 and Figs. 10. It is clear from Figs. 9-10 that the TOA and AOA estimation performances of the first path decrease with the increase of the number of multipath. The estimation performance of this algorithm is optimal under the condition of no multipath, that is, $L_{p}=1$. However, at low SNR of SNR $=5 \mathrm{~dB}$, the estimation accuracy under multipath conditions can still meet the positioning requirements, therefore the algorithm has better adaptability to multipath environment. 


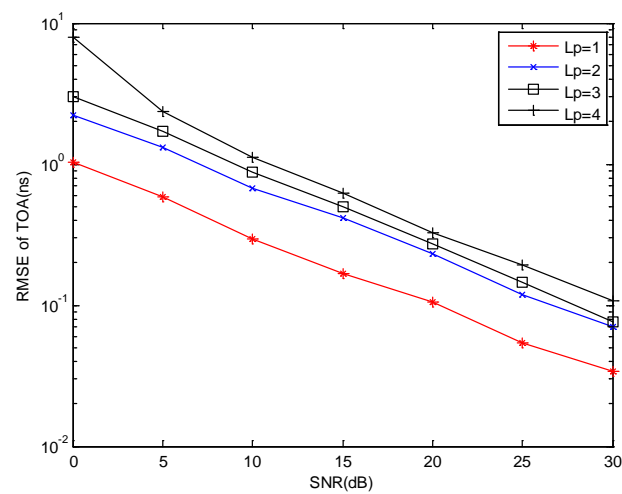

Fig. 9. TOA estimation performance under different paths.

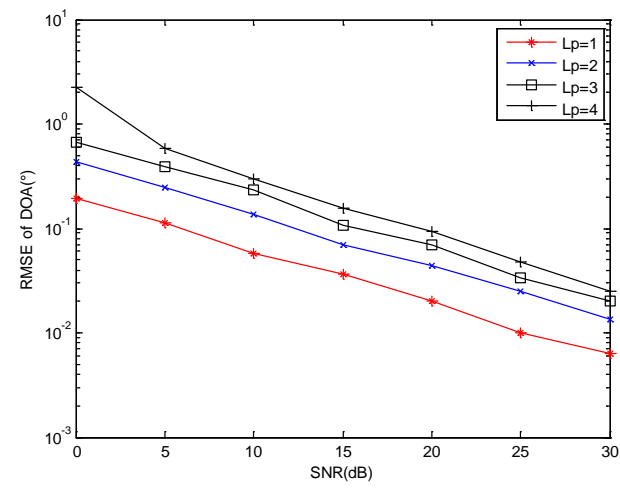

Fig. 10. AOA estimation performance under different paths.

Simulation 5: under the same condition of simulation 1, the proposed algorithm is compared with Root-MUSIC algorithm, 1D MP algorithm [16] [19]. The TOA and AOA RMSE curves of each algorithm's first path are shown in Figs. 11 and Figs. 12, respectively, and compared with the CRB of this model. As is shown in Fig. 11 and Fig. 12, in a single snapshot condition, for the Root-MUSIC algorithm, the decrease of the number of snapshots makes the rank of Vandermonde matrix obtained from eigen-decomposition deficient. Therefore, the estimation performance of the algorithm is always inaccurate.

Meanwhile, because the 2D MP algorithm is adopted in this paper, the performance of the proposed algorithm is obviously superior to that of the algorithm in [16] and [19]. Only from the enhanced matrix (8), we can see that the system bandwidth is enlarged M times for TOA estimation, the equivalent to the number of array elements is $\mathrm{N}$ times larger for AOA estimation. Therefore, in the case of a single snapshot, the estimation accuracy of the proposed algorithm in this paper is much higher than that of the algorithm in [16] and [19].

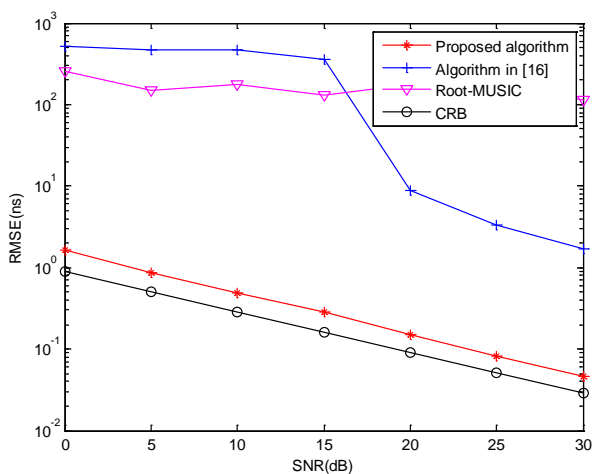

Fig. 11. Performance comparison of different algorithms for TOA estimation.

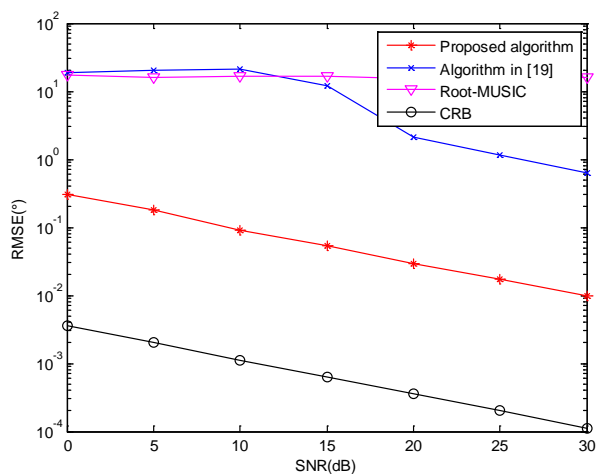

Fig. 12. Performance comparison of different algorithms for AOA estimation.

\section{Conclusion}

Under the condition of the small sample or a single snapshot data, due to the rank loss of the covariance matrix, the parameters estimation performance of wireless positioning system declines sharply. To solve this problem, a joint time delay and angle estimation algorithm 
using 2D MP based on information reconstruction vector is proposed. The detailed construction of the model, the theoretical analysis and the process of CRB derivation are presented, and the joint estimation of high-precision time delay and angle is also achieved in a single snapshot condition. By reconstructing the information parameters and expanding the virtual system bandwidth and virtual array aperture, the joint estimation precision of the time delay and angle of the proposed algorithm is higher, and one receiver can estimate the location of the source target. At the same time, the proposed algorithm does not need to construct covariance matrix and perform pseudo spectral peak search, so the computation speed is fast. The simulation results show that the proposed algorithm, under low SNR, has higher estimation accuracy than Root-MUSIC algorithm and one-dimensional MP algorithm, gains a greater increase in estimation performance at the cost of smaller complexity, and has good adaptability to multipath and fast time-varying environment. Therefore, this algorithm has higher estimation efficiency and certain practical value. In addition, using the unitary transformation can further reduce the computational complexity by the real value solution, and using multiple snapshot data as far as possible can further improve the accuracy of the estimation. These are our next research directions.

\section{Appendix A}

A notation table including the frequently used notations in this paper is as follows.

Table 4. The frequently used notations

\begin{tabular}{cc}
\hline Notation & Definition \\
\hline$N$ & The number of subcarriers \\
$\tau_{i}$ & Propagation delay of the $i$-th multipath signal \\
$\theta_{i}$ & AOA of the $i$-th multipath signal \\
$L_{P}$ & The number of multipath components \\
$M$ & Antenna array elements \\
$a_{i}$ & The complex attenuation of the $i$-th path \\
$n_{m, k}$ & Gauss white noise at the $k$-th subcarrier and the $m$-th antenna element \\
$X_{m, k}$ & Discrete frequency-domain channel response of the $k$-th sampling at the $m$-th antenna element \\
$\boldsymbol{X}$ & Data matrix of the extended spatial $2 \mathrm{D}$ channel frequency response \\
$\boldsymbol{X}_{e}$ & A $K \times(M-K+1)$ enhanced Hankel block matrix \\
$\boldsymbol{X}_{m}$ & A $P \times(N-P+1)$ Hankel matrix \\
$K, P$ & The two window parameters \\
$\boldsymbol{U}_{s}$ & The signal subspace \\
$\boldsymbol{C}$ & The shuffling matrix \\
\hline
\end{tabular}

\section{References}

[1] K. Huo, and J. Zhao, "The development and prospect of the new OFDM radar," Journal of Electronics \& Information Technology, vol. 37, no. 11, pp. 2776-2789, Nov. 2015. Article (CrossRef Link).

[2] Z. Na, Q. Guan, and C. Fu, "Channel model and throughput analysis for LEO OFDM satellite communication system," International Journal of Future Generation Communication \& Networking, vol. 6, no. 6, pp. 109-122, 2013. Article (CrossRef Link).

[3] P. Zhang, Y. Z. Tao, and Z. Zhang, "Survey of several key technologies for 5G, Journal on Communications, vol. 37, no. 7, pp. 15-29, Jul. 2016. Article (CrossRef Link). 
[4] A. Makki, A. Siddig, M. Saad, J. R. Cavallaro, and C. J. Bleakley, "High-resolution time of arrival estimation for OFDM-based transceivers,” Electron. Lett., vol. 51, no. 3, pp. 294-296, Feb. 2015. Article (CrossRef Link).

[5] J. A. D. Peral-Rosado, J. A. López-Salcedo, G. Seco-Granados, F. Zanier, and M. Crisci, “Joint maximum likelihood time-delay estimation for LTE positioning in multipath channels,” EURASIP Journal on Advances in Signal Processing. vol. 2014, no. 33, pp. 1-13, 2014. Article (CrossRef Link).

[6] X. Li, and K. Pahlavan, "Super-resolution TOA estimation with diversity for indoor geolocation," IEEE Trans. Wireless Commun., vol. 3, no. 1, pp. 224-234, Jan. 2004. Article (CrossRef Link).

[7] Z. He, Y. Ma, and R. Tafazolli, "Improved high resolution TOA estimation for OFDM-WLAN based indoor ranging," IEEE Wireless Commun. Lett., vol. 2, no. 2, pp. 163-166, Apr. 2013. Article (CrossRef Link).

[8] H. Ni, G. Ren, and Y. Chang, “A TDOA location scheme in OFDM based WLANs,” IEEE Trans. Consumer Electron., vol. 54, no. 3, pp.1017-1021, Jun. 2008. Article (CrossRef Link).

[9] A. Makki, A. Siddig, M. Saad, J. R. Cavallaro, and C. J. Bleakley, "Indoor localization using 802.11 time differences of arrival,” IEEE Trans. Instrum. Meas., vol. 65, no. 3, pp. 614-623, Mar. 2016. Article (CrossRef Link).

[10] R. Shafin, L. Liu, Y. Li, A. Wang, and J. Zhang, “Angle and delay estimation for 3-D massive MIMO/FD-MIMO systems based on parametric channel modeling," IEEE Trans. Wireless Commun., vol. 16, no. 8, pp. 5370-5383, Aug. 2017. Article (CrossRef Link).

[11] F. Q. Wang, X. F. Zhang, and F. Wang, "Root-MUSIC-based joint TOA and DOA estimation in IR-UWB,” Journal on Communications, vol. 35, no. 2, pp. 137-145, Feb. 2014. Article (CrossRef Link).

[12] B. Zhou, C. Jing, and Y. Kim, "Joint TOA/AOA positioning scheme with IP-OFDM system," Wireless Personal Communication, vol. 75, no. 1, pp. 261-271, 2014. Article (CrossRef Link).

[13] B. Ba, G. Liu, T. Li, Y. Lin, and Y. Wang, "Joint for time of arrival and direction of arrival estimation algorithm based on the subspace of extended hadamard product," Acta Phys. Sin. vol. 64, no. 7, pp. 0784031-0784039, 2015. Article (CrossRef Link).

[14] Y. Hua and T. K. Sarkar, "Matrix pencil method for estimating parameters for exponentially damped/undamped sinusoids in noise,” IEEE Trans. Acoustic, Speech, Signal Process., vol. 38, no. 5, pp.814-824, May 1990. Article (CrossRef Link).

[15] N. Yilmazer, J. Koh, and T. K. Sarkar, "Utilization of a unitary transform for efficient computation in the matrix pencil method to find the direction of arrival,” IEEE Trans. Antennas Propagat., vol. 54, no.1, pp.175-181, Jan. 2006. Article (CrossRef Link).

[16] A. Gaber and A. Omar, "A study of TDOA estimation using matrix pencil algorithms and IEEE 802.11ac," in Proc. of IEEE Proc. Ubiquitous Positioning, Indoor Navigat., Location Based Service (UPINLBS), pp. 1-8, Oct. 2012. Article (CrossRef Link).

[17] Y. Hua, "Estimating two-dimensional frequencies by matrix enhancement and matrix pencil," IEEE Trans. Signal Process., vol. 40, no. 9, pp. 2267-2280, Sep. 1992. Article (CrossRef Link).

[18] N. Yilmazer, and T. K. Sarkar, "2-D unitary matrix pencil method for efficient direction of arrival estimation,” Digital Signal Process., vol. 16, pp.767-781, Jan. 2006. Article (CrossRef Link).

[19] R. Ding, Z. H. Qian, and X. Wang, "UWB Positioning System Based on Joint TOA and DOA Estimation,” Journal of Electronics \& Information Technology, vol. 32, no. 2, pp. 313-317, Feb. 2010. Article (CrossRef Link).

[20] Y. Wang, J. W. Chen, and Z. Liu, "Comments on estimation of frequencies and damping factors by Two-Dimensional ESPRIT type methods,” IEEE Trans. Signal Process., vol. 53, no. 8, pp. 3348-3349, Aug. 2005. Article (CrossRef Link).

[21] M. C. Vanderveen, A.-J. van der Veen, and A. Paulraj, "Estimation of multipath parameters in wireless communications,” IEEE Trans. Signal Process., vol. 46, no. 3, pp. 682-690, Mar. 1998. Article (CrossRef Link).

[22] P. Stoica and A. Nehorai, "Music, maximum likelihood, and cramer-rao bound," IEEE Trans. Acoust., Speech, Signal Process., vol. 37, no. 5, pp. 720-741, May 1989. Article (CrossRef Link). 


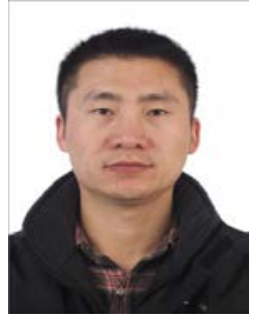

Haiwen Li received the B.S. degree in network engineering from Xi'an Communication Institute, Xi' an, China, in 2003 and the M.S. degree from Chongqing University of Posts and Telecommunications, China, in 2010. He is currently pursuing the Ph.D. degree in information and communication engineering at China National Digital Switching System Engineering and Technological Research Center, Zhengzhou, China. His research interests include image processing, mobile communication technology, wireless positioning technology and digital signal processing.

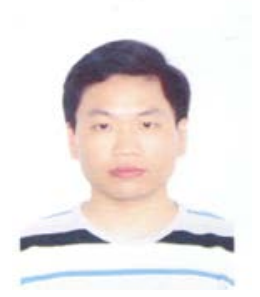

Xiukun Ren received his BS and MS degrees in mobile communication and wireless positioning technology from Information Engineering University, Henan, China, in 2000, and 2007, respectively. Since 2000, he has been working in National Digital Switching System Engineering and Technological Research Centre, Zhengzhou, China, where he is now an associate professor. His main research interests are wireless communication and wireless positioning technology.

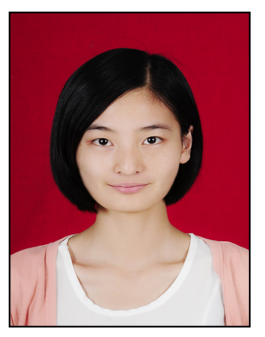

Ting Bai received her BS, MS degrees in information engineering university, Henan, China, in 2015 and 2017, respectively. Since 2017, she is now working toward her PhD degree at China National Digital Switching System Engineering and Technological Research Center, Zhengzhou, China. Her main research interest is OFDM MIMO radar waveform design.

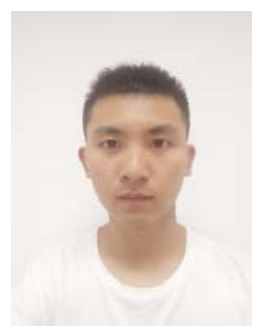

Long Zhang received his B.S. degree in information confrontation technology from Harbin Engineering University, Harbin, China, in 2012-2016.From 2016 to now, he is studying for M.S. degree in Information and Communication Engineering at China National Digital Switching System Engineering and Technological Research Center, Zhengzhou, China. His research interests include indoor wireless location technology, fusion indoor location and so on. 